\title{
Hamiltonian Dynamics of Darwin Systems
}

\author{
Alexander V. Shapovalov \\ Tomsk State University, Tomsk, RUSSIA \\ E-mail:shpv@phys.tsu.tomsk.su \\ Eugene V. Evdokimov \\ Research Institute of Biology and Biophysics, Tomsk, RUSSIA \\ E-mail:evd@biobase.tsu.tomsk.su
}

\begin{abstract}
We present a Hamiltonian approach for the wellknown Eigen model of the Darwin selection dynamics. Hamiltonization is carried out by means of the embedding of the population variable space, describing behavior of the system, into the space of doubled dimension by introducing additional dynamic variables. Besides the study of the formalism, we try to interpret its basic elements (phase space, Hamiltonian, geometry of solutions) in terms of the theoretical biology. A geometric treatment is given for the considered system dynamics in terms of the geodesic flows in the Euclidean space where the population variables serve as curvilinear coordinates.

The evolution of the distribution function is found for arbitrary distributed initial values of the population variables.

PACS: 87.10.+e
\end{abstract}

Keywords: Hamiltonian dynamics, Darwin systems 


\section{INTRODUCTION}

Methods of Hamiltonian and Lagrangian analysis are extensively applied during last decades in various fields of theoretical and mathematical physics. These methods, however, have not been widely used until now in mathematical theory of biological and ecological systems which foundations originated from the works of the 10-30-es by A. Lotka, V. Volterra, J. Haldane and R. Fisher.

The basic obstacle, by our view, is that the mathematical models of biological subjects, being open systems in their essence, are not supposed to be Hamiltonian ones from the outset. On the other hand, broad potentialities may appear for biological systems to analyze some aspects (of both principle and technical character) of their dynamics, if the system admits Hamiltonization in a sense.

In the present paper we suggest a Hamiltonian form for the wellknown Eigen model of the Darwin selection dynamics. To construct the Hamiltonian formalism, we extent the space of population variables introducing additional degrees of freedom. As a result the behavior of the system is described in terms of the space with doubled dimension if compared to the original one. Besides the study of the formalism, we try to interpret its basic elements (phase space, Hamiltonian, geometry of solutions) in terms of the theoretical biology. A geometric treatment is given for the considered system dynamics in terms of the geodesic flows in the Euclidean space where the population 
variables serve as curvilinear coordinates.

The notion of a Darwin system (DS) as a formal object in theoretical biology had been introduced by M. Eigen to deduce laws of living system evolution from the principles of theoretical physics and chemistry.

By definition, the DS is an open system, which is constituted of coupling units of different species (genotypes) self-copying with a small number of errors (convariantly self-reduplicating according to Timofeev-Resovskii nomenclature [1]). The coupling units utilize a substance and free energy of an external nutrient supply [2].

The Darwin selection phenomenon occurs in such systems under the following necessary conditions: the stability of system organization (i.e. the total quantity of units of all species should conserve) or the stability of feed component influx. Both of these constraints imply the flow through the system. A simple experimental model of the DS (chemostat, turbidostat, etc.) can be a system where viruses and bacteria are defined as convariantly self-reduplicating units (see, for example, [3]).

The following system of differential equations proposed by M. Eigen to describe the DS evolution:

$$
\dot{x}_{i}=x_{i}\left(A_{i} Q_{i}-D_{i}\right)+\sum_{j \neq i}^{N} w_{j i} x_{j}-\Phi_{i},
$$

here $i, j, k=1, \ldots, N ; N$ is a species quantity of self-reduplicating units (genotypes) in the system; $x_{i}$ is a specific quantity (concentration) of the $i$-th species; $A_{i}\left(D_{i}\right)$ is a specific reproduction (death) velocity of the $i$-th species; 
$Q_{i}$ is a parameter of the reduplication quality of the $i$-th species (takes the values from 0 to 1$) ; w_{j i}$ is a specific velocity of the error reduplication of the $j$-th species into the $i$-th one; $\Phi_{i}$ is a dilution parameter which usually equals to $D x_{i}$. Here $D$ is a dilution flow rate.

Further, as it has been shown by M. Eigen [2], the system (四) can be put in a simpler form

$$
\dot{y}_{i}=y_{i}\left(\mu_{i}(\mathbf{S})-D\right)
$$

using obvious relations between $A_{i}, Q_{i}, w_{j i}$ and by introducing new population variables $\left(y_{i}\right)$, being related to the so called "quasispecies" which are described by certain combinations of original variables $\left(x_{i}\right)$. Here $i, j, k=1, \ldots, N ; N$ is a (constant) quasispecies quantity in the system; $y_{i}$ has a meaning of a specific quantity (concentration) of the $i$-th quasispecies; $\mathbf{S}=\left(S_{1}, \ldots, S_{f}\right)$ are the concentrations of the external nutrient supply components; $\mu_{i}(\mathbf{S})$ is a generalized Maltuzian parameter which has a meaning of the reproduction specific velocity of the $i$-th quasispecies and depends on $\mathbf{S}$. The $\mu_{i}(\mathbf{S})$ is an algebraic combination of all $A_{k}, D_{k}, w_{j k}$ in which $A_{i}$ makes a major contribution. The $\mu_{i}(\mathbf{S}), D$ have dimension of inverse time. The "quasispecies" introduced by M. Eigen as new population variables have a clear biological sense of clones, systems of organisms with a prevalence of the determined genotype in the system and small admixtures of some other genotypes connected with the leading one by the mutation and recombination transitions [2]. 
There are two commonly recognized types of DS under the constraints imposed above on the system (回). Recall the definitions of these types.

Definition I.1 A Darwin system is referred to as DS with stable organization (DSSO) if $\sum_{i=1}^{N} y_{i}$ and $\mathbf{S}$ are constant. A Darwin system with $D=$ const is referred to as DS with a stable flow (DSSF).

It is known that the representation of the system (2) in terms of relative variables having a meaning of shares (probabilities) of the quantities [田] transforms (2) into the classical form of the Fisher system of equations which describes the Darwin selection dynamics in the panmictic populations under the special requirement for the fitness function [5]. The system (2]) is also valid for description of interspecies competition for the grow-determining substrate when there is no migration. In view of significance of the systems (回),(目) in biology and ecology they are investigated in a large number of works since the paper [5] has appeared. So we touch upon some of these publications relevant for the present paper.

An exact solution of (2) is known only for $\mu_{i}=$ const [6], a general form of approximate solution is found by Jones [7] for the $\mu_{i}$ changing in time. A general review of the properties of system (2) in the Fisher modification is given in [8], [9]. Stability of the stationary solutions of (2]) is analyzed in detail by Pykh [10]. Feistel and Ebeling have studied behavior of the Eigen-Fisher system on the adaptive Right's landscape and shown that in a number of cases the system can be represented in the canonical gradient 
form [团, [11]. Isida [12] investigated non-equilibrium thermodynamics of the systems described by the Eigen equations. As is known, there are two different approaches to describe Darwin, Fisher and reducible to them Volterra systems by means of extremal principles. The first (teleological) approach assumes to extremalize of a functional having a certain biological sense as a finite aim of the evolution. For example, it might be maximum of an average population fitness [5], or maximum of an average productivity [2], or maximum of a reproductive potential [\&] etc. The second approach assumes to achieve the extremum of some function of dynamic variables in each moment of time. Thus, in reference [13] it is shown that the requirement for Kulback's measure to have a maximum value leads to the relations which are the solutions of (2) under the special conditions imposed on the system in the approximation of the DSSO. As a next example we refer to the work [9] where variational form of the Eigen-Fisher dynamics in a special case is built up by introduction of a metrics of a Riemann space. Despite the fact that the dynamics of the Eigen-Fisher type systems are managed to represent in the form of extremal principles in some special cases, the problem stays far from complete solution as it has been explained in [8].

The Hamiltonian form of the DS dynamic equations, being proposed in the present work, leads to the conventional variational principle, which is habitual in mechanics. 


\section{DS WITH A STABLE ORGANIZATION}

In accordance with def.[.], Eqs. (22) assume the following form for the DSSO case:

$$
\dot{y}_{i}=y_{i}\left(\mu_{i}-\sum_{j=1}^{N} y_{j} \mu_{j} / \sum_{j=1}^{N} y_{j}\right), \quad \mu_{i}=\text { const. }
$$

The dimensionless variables $p_{i}=y_{i} / \sum_{j=1}^{N} y_{j}$ have a meaning of shares (probabilities) of the quantities. Taking into account that $\sum_{j=1}^{N} y_{j}=$ const, we derive from (지) for $p_{i}$ the equations:

$$
\dot{p}_{i}=p_{i}\left(\mu_{i}-\sum_{j=1}^{N} p_{j} \mu_{j}\right)
$$

If $p_{N}$ is eliminated solving the constraint $\sum_{j=1}^{N} p_{j}=1$, one can rewrite (田) as follows:

$$
\dot{p}_{i}=p_{i}\left(a_{i}-\sum_{j=1}^{N-1} p_{j} a_{j}\right)
$$

Here and up to the end of this section we assume: $i, j, k=1, \ldots, N-1, a_{i}=$ $\mu_{i}-\mu_{N}, \mu_{N}$ is a Maltuzian parameter of $N$-th quasispecies taken as a gauge one, for example, by the criterium of minimal value of $\mu_{i}$. Next, to simplify (5) we introduce the following variables:

$$
z_{i}=\log \left[p_{i} /\left(1-\sum_{j=1}^{N-1} p_{j}\right)\right]
$$

Accounting the condition $1-\sum_{j=1}^{N-1} p_{j}=p_{N}$, the new variables $z_{i}$ might be called as informational ones, inasmuch as they reflect, in a spirit of the Shannon's theory [14], a quantity of information for every independent degree of freedom co-ordinated to the eliminated $N$-th variable. 
Consider variables (6) as coordinates of a manifold $\mathcal{A}$ which naturally can be named as an information one. A state of the system in $\mathcal{A}$ is characterized by a point $\mathbf{z}=\left(z_{1}, z_{2}, \ldots, z_{N-1}\right)$. The system dynamics $\mathbf{z}(t)$ is described by a velocity vector $\dot{\mathbf{z}}(t)$. The system (5) in the variables $\mathbf{z}$ takes the simple form,

$$
\dot{z}_{i}=a_{i}
$$

and has the general solution

$$
z_{i}(t)=z_{i_{0}}+a_{i} t
$$

Hence, evolution of the DSSO in the "information" variables (6]) is a uniform rectilinear motion. Taking "mass" to be a unit, we can conceive that the $a_{i}$ is the $i$-th component of the momentum. In accordance with Hamiltonian formalism, we will interpret $\left(z_{i}\right)$ and $\left(a_{i}\right)$ as the position and momentum variables, respectively, in a $2(N-1)$-dimensional phase space $\mathcal{M}$ of the system. Introducing Hamiltonian of the system

$$
h=\frac{1}{2} \sum_{j=1}^{N-1} a_{j}^{2},
$$

we can write down the Hamiltonian form of the system $(\square)$ as follows:

$$
\left\{\begin{array}{c}
\dot{z}_{i}=\partial h / \partial a_{i}=a_{i}, \\
\dot{a}_{i}=-\partial h / \partial z_{i}=0 .
\end{array}\right.
$$

In the framework of the considered Hamiltonian approach we can state the following variational principle for the DSSO dynamics. Lagrange function of the system (9),$l=\sum_{j=1}^{N-1} a_{j} \dot{z}_{j}-h$, after the momentum variables $\left(a_{i}\right)$ eliminating, takes the form: 


$$
l(z, \dot{z})=\frac{1}{2} \sum_{j=1}^{N-1} \dot{z}_{j}^{2}
$$

The minimal action principle is:

$$
A=\int_{0}^{t} l(z, \dot{z})(\tau) d \tau, \quad \delta A=0
$$

The variational principle $([0]),([1])$ leads to the following treatment of the DS dynamics:

a DSSO evolutes in the information manifold $\mathcal{A}$ in such a way which minimalizes the squared norm of the respective velocity of variation of information in the system at the considered time interval.

Canonical form of the dynamic equations (5) is found from the canonical transformation $\left(z_{i}, a_{i}\right) \rightarrow\left(p_{i}, r_{i}\right)$, where $r_{i}$ are additional variables canonically conjugate to $p_{i}$. This transformation is:

$$
\left\{\begin{array}{l}
p_{i}=e^{z_{i}} /(1+\Delta), \quad \Delta \equiv \sum_{j=1}^{N-1} e^{z_{j}} \\
r_{i}=(1+\Delta)\left[a_{i} e^{-z_{i}}+\sum_{j=1}^{N-1} a_{j}\right] .
\end{array}\right.
$$

The inverse transformation:

$$
\left\{\begin{array}{c}
e^{z_{i}}=p_{i} /(1-\Omega), \quad \Omega \equiv \sum_{j=1}^{N-1} p_{j}, \\
a_{i}=p_{i}\left(r_{i}-w\right), \quad w=\sum_{j=1}^{N-1} r_{j} p_{j} .
\end{array}\right.
$$

Eqs. (9), (12),(13) lead to the original system (5) to be supplemented by the equations for $r_{i}$ :

$$
\left\{\begin{array}{c}
\dot{p}_{i}=p_{i}\left[p_{i}\left(r_{i}-w\right)-\sum_{j=1}^{N-1} p_{j}^{2}\left(r_{j}-w\right)\right] \\
\dot{r}_{i}=r_{i} \sum_{j=1}^{N-1} p_{j}^{2}\left(r_{j}-w\right)-p_{i}\left(r_{i}-w\right)^{2}
\end{array}\right.
$$


Using (8), ([2]),([3]) we obtain the solution of ([4]) in the form:

$$
\begin{gathered}
p_{i}(t)=p_{0 i} \frac{\exp \left[p_{0 i}\left(r_{0 i}-w_{0}\right) t\right]}{1-\Omega_{0}+\sum_{k=1}^{N-1} p_{0 k} \exp \left[p_{0 k}\left(r_{0 k}-w_{0}\right) t\right]}, \\
r_{i}(t)=\left\{r_{0 i} \exp \left(-p_{0 i}\left(r_{0 i}-w_{0}\right) t\right)+w_{0}\left[1-\exp \left(-p_{0 i}\left(r_{0 i}-w_{0}\right) t\right)\right]\right\} \times \\
\left\{1-\Omega_{0}+\sum_{k=1}^{N-1} p_{0 k} \exp \left(p_{0 k}\left(r_{0 k}-w_{0}\right) t\right)\right\} .
\end{gathered}
$$

Here $p_{0 k}=\left.p_{k}(t)\right|_{t=0}, r_{0 k}=\left.r_{k}(t)\right|_{t=0}$ are the initial conditions for the system ([4) $, w_{0}=\sum_{k=1}^{N-1} p_{0 k} r_{0 k}, \Omega_{0}=\sum_{k=1}^{N-1} p_{0 k}$. Expressions $r_{0}(t, p, r), p_{0}(t, p, r)$ are obtained from ([5), ([6]) by the substitution: $p_{0 i} \leftrightarrow p_{i}, r_{0 i} \leftrightarrow r_{i}, t \rightarrow-t$. The system (14) is the Hamiltonian one in terms of the canonical coordinates $\left(p_{i}, r_{i}\right)\left(p_{i}\right.$ are the position variables and $r_{i}$ are the momentum ones) with the Hamiltonian

$$
h=\frac{1}{2} \sum_{j=1}^{N-1} p_{j}^{2}\left(r_{j}-w\right)^{2}
$$

The respective dynamics can be represented in terms of the geodesic flows of the $(N-1)$ - dimensional Euclidean space where $p_{i}$ serves as curvilinear coordinates and $z_{i}$ are the Cartesian ones. To impart tensor nature to the notations, let $z_{i} \rightarrow z^{i}, p_{i} \rightarrow p^{i}$ leaving subscripts at $a_{i}, r_{i}$. The Hamilton function (1T7) can be rewritten as

$$
h=\frac{1}{2} \sum_{j=1}^{N-1} g^{i j}(p) r_{i} r_{j}
$$

Here $g^{i j}(p)$ are the contravariant components of the metric tensor in the coordinates $\left(p_{i}\right)$ : 


$$
g^{i j}(p)=\sum_{k=1}^{N-1} \frac{\partial p^{i}}{\partial z^{k}} \frac{\partial p^{j}}{\partial z^{k}}=\left(p^{i}\right)^{2} \delta^{i j}-\left[\left(p^{i}\right)^{2} p^{j}+p^{i}\left(p^{j}\right)^{2}\right]+R^{2} p^{i} p^{j}
$$

where $R^{2}=\sum_{k=1}^{N-1}\left(p^{i}\right)^{2}$. The covariant components are:

$$
g_{i j}(p)=\sum_{k=1}^{N-1} \frac{\partial z^{k}}{\partial p^{i}} \frac{\partial z^{k}}{\partial p^{j}}=\frac{\delta_{i j}}{\left(p^{i}\right)^{2}}+\frac{1}{p^{i}(1-\Omega)}+\frac{1}{p^{j}(1-\Omega)}+\frac{N-1}{(1-\Omega)^{2}},
$$

$$
\operatorname{det}\left(g^{i j}(p)\right)=(1-\Omega)^{2} \Pi_{j=1}^{N-1}\left(p^{j}\right)^{2},
$$

$\sum_{k=1}^{N-1} g^{i k} g_{k j}=\delta_{j}^{i}, \quad\left(\delta_{i j}, \delta^{i j}, \delta_{j}^{i}\right.$ are the Kronecker deltas $)$. The Lagrange function,

$$
l=\sum_{k=1}^{N-1} \dot{p}^{k} r_{k}-h=\frac{1}{2} \sum_{k, l=1}^{N-1} g_{k l}(p) \dot{p}^{k} \dot{p}^{l}
$$

defines the Euler-Lagrange equations,

$$
d / d t\left(\partial l / \partial \dot{p}^{j}\right)-\partial l / \partial p^{j}=0
$$

which take the form of geodesic equations:

$$
\frac{d^{2} p^{j}}{d t^{2}}+\sum_{k, l=1}^{N-1} \Gamma_{k l}^{j}(p) \dot{p}^{k} \dot{p}^{l}=0
$$

where $\Gamma_{k l}^{j}(p)=\frac{1}{2} \sum_{s=1}^{N-1} g^{j s}\left(\partial g_{k s} / \partial p^{l}+\partial g_{l s} / \partial p^{k}-\partial g_{k l} / \partial p^{s}\right)$ are the Cristoffel symbols.

Let us point out that the coordinates $p_{i}$ are nonorthogonal ones due to nondiagonality of the metrics in these coordinates.

The DS trajectories $p_{i}(t)$ are usually mapped as curved lines in the coordinates $p_{i}$ supposing them to be orthogonal Cartesian ones (see, for example, 
[2]), whereas this motion can be represented as straight lines in the Cartesian coordinates $z_{i}$. Twisting of the trajectory with respect to $\left(p_{i}\right)$ is the result of the curvilinearity of these coordinates. The existence of the coordinates $\left(z_{i}\right)$, where the DSSO dynamics is represented by a uniform rectilinear motion, illustrates the absence of a coupling between quasispecies and any external factors in the course of the Darwin selection process. From this standpoint, the DSSO can serve as a biology-theoretic analogue of an inertial reference system in the Newtonian mechanics.

Let us note that the $a_{i}$ give rise to an Abelian algebra of integrals of the system (14) which is integrable by Liouville with respect to the conventional Poisson brackets $\{f, g\}=\sum_{j=1}^{N-1}\left(\partial f / \partial p_{j} \partial g / \partial r_{j}-\partial g / \partial p_{j} \partial f / \partial r_{j}\right)$. The system

$$
\left\{\begin{array}{l}
d p_{i} / d \alpha=\partial a_{q} / \partial r_{i}=p_{q}\left(\delta_{q i}-p_{i}\right), \\
d r_{i} / d \alpha=-\partial a_{q} / \partial p_{i}=-\delta_{i q}\left(r_{q}-w\right)+p_{q} r_{i}
\end{array}\right.
$$

defines a flow generated by $a_{q}$ in the phase space $\mathcal{M}$ which leaves Eqs. ([4]) invariant, $\alpha$ is a group parameter. Integration gives:

$$
\begin{aligned}
& p_{q}(\alpha)=p_{q}(0) e^{\alpha} V(\alpha)^{-1} ; \\
& p_{i}(\alpha)=p_{i}(0) V(\alpha)^{-1}, \quad i \neq q ; \\
& r_{q}(\alpha)=e^{-\alpha} V(\alpha)^{2}\left[r_{q}(0)+W\left(e^{\alpha}-1\right) V(\alpha)^{-1}\right] ; \\
& r_{i}(\alpha)=r_{i}(0) V(\alpha), \quad i \neq q .
\end{aligned}
$$

Here $V(\alpha) \equiv 1+\left(e^{\alpha}-1\right) p_{q}(0), W \equiv \sum_{j \neq q} r_{j}(0) p_{j}(0), p_{i}(0), r_{i}(0)$ are initial conditions for (22). Let us note, that the variables $p_{i}$ in (23) transform independently of $r_{i}$ so that one can directly use (223) for the original system 
(可)

In conclusion of this section we consider DSSO dynamics with less number of constraints then in the def.[.]. Let us retire only the requirement of constant of total quantity of all species in the system and remove the restrictions on the nutrient supply concentration and on some other parameters of the medium, for example, on a temperature. This leads for $\mu_{i}$ to be certain functions of time. Since $\mu_{i}$ are not involved in the transformation from the original variables to the "information" ones, Hamiltonian form of the DS dynamics can be thereby constructed in the considered case too.

Let $f_{i}$ be the first derivative of $a_{i}, \dot{a}_{i}=f_{i}$. Since $a_{i}$ serve as momentum variables in the DS dynamics, then the derivatives of $f_{i}$ can be considered as components of a force. It is easy to show that the Hamiltonian of the considered system takes the form:

$$
h(z, a)=\sum_{i=1}^{N}\left(\frac{1}{2} a_{i}^{2}-z_{i} f_{i}\right) .
$$

Respectively, Hamiltonian form of Eqs. (9) is:

$$
\left\{\begin{array}{c}
\dot{z}_{i}=\partial h / \partial a_{i}=a_{i}, \\
\dot{a}_{i}=-\partial h / \partial z_{i}=f_{i} .
\end{array}\right.
$$

The Hamiltonian (24) plays a role of an "energy" in the Darwin dynamics if functions $f_{i}$ are constant in time. The second term in (24) has a sense of a "potential energy". The DS dynamics described by the system (25) is a motion in the field of the "force" $f_{i}$. The additional "potential" term leads to the variational principle $([10),([1])$ with the modified Lagrangian: 


$$
l=\sum_{j=1}^{N} \dot{z}_{i} a_{i}-h=\sum_{j=1}^{N}\left(\frac{1}{2} \dot{z}_{i}^{2}+z_{j} f_{j}\right) .
$$

\section{DS WITH A STABLE FLOW}

To provide Eqs. (回) to be a complete system in the case of DSSF (in the sense of def.[.]), one has to define explicitly the functions $\mu_{i}(\mathbf{S})$ and to extend the system introducing equations for $\mathbf{S}$. Here and further $i, j, k=1, \ldots, N$.

The wellknown Monod function [15] is commonly recognized for DSs. It depends on one independent variable $S$ which has a meaning of concentration of the substrate being in physiological minimum (growth limiting substrate). That is the concentration vector $\mathbf{S}$ is reduced to one component $S$. The dynamic system in this case takes the form:

$$
\begin{aligned}
\dot{y}_{i} & =y_{i}\left(\mu_{i}(S)-D\right), \\
\dot{S} & =D\left(S_{0}-S\right)-\sum_{j=1}^{N} y_{j} \mu_{j}, \\
\mu_{i} & =m_{i} S /\left(K_{i}+S\right),
\end{aligned}
$$

where $m_{i}$ is a maximal reproduction specific velocity, $K_{i}$ is a saturation parameter of $i$-th quasispecies, $m_{i}, K_{i}=$ const, $S$ is a concentration of the growth limiting substrate in the system, $S_{0}$ is its concentration in the external flow.

There is no apparent way to solve the system (27) and to hamiltonize it under arbitrary values of parameters.

For the further analysis of this system let us take into account some qualitative peculiarities of its dynamics which are known from experimental data 
and numerical simulations [15], [17].

The system dynamics in general case is characterized by two basic stadium:

the first one is an initial transient with a period of order $2 / D \div 4 / D$ where the function $S(t)$ varies strongly and nonlinearly;

the second one is a quasistationary behavior in the interval $4 / D<t<\tau$ where $\tau$ is an observation time of the system. $S(t)$ can be approximated in this case by a linear function in this range up to 3-7\%. Let

$$
S(t)=\bar{S}+b\left(t-\frac{1}{2} \tau\right) .
$$

Here $\bar{S}$ is an average value of the $S(t)$ during the observation time $\tau$ of the system, $b$ is an average velocity of the concentration variation of the ratedetermining substrate in the system.

Moreover, $\mu_{i}(S)$ can be also approximated by a linear function in the range of the quasistationary behavior where $S(t)<K_{i}$.

Expanding Monod function (27) in a neighborhood of the point $S=\bar{S}$, we have in the second order:

$$
\begin{aligned}
& \mu_{i}(S)=\tilde{\alpha}_{i}+\tilde{\beta}_{i} S, \\
& \tilde{\alpha}_{i}=\mu_{i}(\bar{S})-\bar{S}\left(\partial \mu_{i}(\bar{S}) / \partial \bar{S}\right)=m_{i} \bar{S}^{2} /\left(K_{i}+\bar{S}\right)^{2}, \\
& \tilde{\beta}_{i}=\partial \mu_{i}(\bar{S}) / \partial \bar{S}=m_{i} K_{i} /\left(K_{i}+\bar{S}\right)^{2} .
\end{aligned}
$$

With the approximations above, the DSSF dynamics in the quasistationary stadium is described by the following equations:

$$
\dot{y}_{i}=y_{i}\left(\alpha_{i}+\beta_{i} \cdot t\right),
$$


where $\alpha_{i}=\tilde{\alpha}_{i}+\tilde{\beta}_{i}\left(\bar{S}-\frac{1}{2} b \tau\right)-D, \beta_{i}=b \cdot \tilde{\beta}_{i}$. It is easily to give a Hamiltonian form for the system (28). Choosing canonical variables in a $2 N$-dimensional phase space as

$$
z_{i}=\log y_{i}, \quad a_{i}=\alpha_{i}+\beta_{i} \cdot t
$$

and the Hamiltonian

$$
h(z, a)=\sum_{i=1}^{N}\left(\frac{1}{2} a_{i}^{2}-z_{i} \beta_{i}\right)
$$

we arrive to the Hamiltonian form of Eqs. (28):

$$
\left\{\begin{array}{c}
\dot{z}_{i}=\partial h / \partial a_{i}=a_{i}, \\
\dot{a}_{i}=-\partial h / \partial z_{i}=\beta_{i} .
\end{array}\right.
$$

Note, that the hamiltonian form of the DSSF (29) is similar to the DSSO case (25) and it describes uniformly accelerated rectilinear motion in space of variables $\left(z_{i}\right)$ in a constant and homogeneous external field with the potential $U(z)=-\sum_{j=1}^{N} \beta_{j} z_{j}$ determining a constant "force" $\mathbf{F}=\beta$. The Lagrange function is:

$$
l=\sum_{j=1}^{N} \dot{z}_{i} a_{i}-h=\sum_{j=1}^{N}\left(\frac{1}{2} \dot{z}_{i}^{2}+z_{j} \beta_{j}\right) .
$$

The variational principle has the form ([1]) where $l(z, \dot{z})$ is of the form (B0]). Canonical transformation $\left(z_{i}, a_{i}\right) \rightarrow\left(y_{i}, r_{i}\right)$

$$
\left\{\begin{aligned}
z_{i} & =\log y_{i} \\
a_{i} & =y_{i} r_{i}
\end{aligned}\right.
$$

allows to rewrite (28) in Hamiltonian form, 


$$
\left\{\begin{array}{l}
\dot{y}_{i}=\partial h / \partial r_{i}=y_{i}^{2} r_{i} \\
\dot{r}_{i}=-\partial h / \partial y_{i}=-y_{i} r_{i}^{2}+\beta_{i} y_{i}^{-1}
\end{array}\right.
$$

with the Hamiltonian

$$
h(y, r)=\sum_{i=1}^{N}\left(\frac{1}{2} y_{i}^{2} r_{i}^{2}-\beta_{i} \log y_{i}\right)=\sum_{i, j=1}^{N} \frac{1}{2} g^{i j}(y) r_{i} r_{j}+U(y),
$$

$U(y)=-\sum_{i=1}^{N} \beta_{i} \log y_{i}$. The solution of the system (32) with the initial conditions $\left.y_{i}(t)\right|_{t=0}=y_{i 0},\left.r_{i}(t)\right|_{t=0}=r_{i 0}$ takes the form :

$$
\begin{aligned}
& y_{i}(t)=y_{i 0} \exp \left(y_{i 0} r_{i 0} t+\frac{1}{2} \beta_{i} t^{2}\right), \\
& r_{i}(t)=\frac{y_{i 0} r_{i 0}+\beta_{i} t}{y_{i 0} \exp \left(y_{i 0} r_{i 0} t+\frac{1}{2} \beta_{i} t^{2}\right)} .
\end{aligned}
$$

Expressions of $y_{0}(t, y, r), r_{0}(t, y, r)$ as in the case of (15), (15) are obtained from (33) by substitution: $y_{0 i} \leftrightarrow y_{i}, r_{0 i} \leftrightarrow r_{i}, t \rightarrow-t$.

Redenoting $y_{i} \rightarrow y^{i}$, we can write down contravariant and covariant components of the metrics as follows: $g^{i j}(y)=\delta^{i j}\left(y^{i}\right)^{2}, g_{i j}(y)=\delta_{i j}\left(y^{i}\right)^{-2}$, respectively. The Lagrange function, $l=\sum_{k=1}^{N} \dot{y}^{k} r_{k}-h$, and the Euler-Lagrange equations (21) take here the form:

$$
\begin{gathered}
l=\frac{1}{2} \sum_{i, j=1}^{N} g_{i j}(y) \dot{y}^{i} \dot{y}^{j}-U(y) \\
\frac{d^{2} y^{j}}{d t^{2}}+\sum_{k, l=1}^{N} \Gamma_{k l}^{j}(y) \dot{y}^{k} \dot{y}^{l}=-\sum_{k=1}^{N} g^{j k}(y) \frac{\partial U(y)}{\partial y^{k}}
\end{gathered}
$$

respectively, where $\Gamma_{k l}^{j}(y)$ are the Christoffel symbols related to the metrics $g_{i j}(y)$. 


\section{DISTRIBUTION FUNCTION OF INITIAL DATA FOR DS}

Hamiltonian form of the DS dynamics allows one to investigate evolution of an initial data distribution. Consider this problem for the DSSO case in more detail. Let initial data for the DSSO are given in a domain $\mathcal{O}$ of phase space $\mathcal{M}$ with a distribution function $f_{0}(p, r), f_{0}(p, r) \geq 0$, $\int_{\mathcal{O}} f_{0}(p, r) d p d r=1$. The problem of initial data indeterminancy arises for the Darwin systems due to natural restrictions on the exactness of measurements and Poisson character of initial values distribution of separate genotypes quantity. Consider the evolution of the distribution function $f(t, p, r)$ under the condition that

$$
\left.f(t, p, r)\right|_{t=0}=f_{0}(p, r) .
$$

Supposing that the randomness is brought in the DS behavior only in the form of random distribution of initial data and further DS evolution is determined and is subjected to the Hamiltonian equations above, we deduce that the distribution remains constant on the phase trajectories on account of conservative character of the Hamiltonian dynamics. In other words, the distribution function $f(t, p, r)$ obeys the Liouville equation:

$$
\frac{\partial f}{\partial t}+\{f, h\}=0
$$

The solution of (35) with the initial condition (34) is obtained by substitution

of the expressions $p_{0}(t, p, r), r_{0}(t, p, r)$ inverting formulas (15), (16) into the initial function $f_{0}$ : 


$$
f(t, p, r)=f_{0}\left(p_{0}(t, p, r), r_{0}(t, p, r)\right) .
$$

As an illustration let us consider more specifically the simplest case of phase space $N=2$ when a point of phase space $\mathcal{M}$ is defined by one coordinate $(p)$ and one momentum $(r)$ variables. In this case $p_{0}(t, p, r), r_{0}(t, p, r)$ are written down in the form:

$$
\begin{array}{r}
p_{0}(t, p, r)=\frac{1}{2}[1-\tanh (t \cdot \theta+\delta)], \\
r_{0}(t, p, r)=8 \theta \cosh ^{2}(t \cdot \theta+\delta),
\end{array}
$$

where $\theta=\frac{1}{2} r p(1-p), \delta=\frac{1}{2} \log \frac{(1-p)}{p}$.

$$
f(t, p, r)=f_{0}\left(\frac{1}{2}[1-\tanh (t \cdot \theta+\delta)], 8 \theta \cosh ^{2}(t \cdot \theta+\delta)\right) .
$$

Eq. (37) allows one to obtain time-dependent distribution function of $p$ :

$$
f(t, p)=\int f(t, p, r) d r
$$

Evolution of the distribution function can be inferred from the transformation of equiprobabilistic surfaces which are obtained from equation $f(t, p, r)=$ const in different moments of time $t$.

Similar results can be easily obtained in the case of DSSF. Evolution of the distribution function is given by (36) in which it is necessary to substitute the equations $y_{0}(t, y, r), r_{0}(t, y, r)$ inverting (33).

\section{CONCLUSION}

Hamiltonian form of the dynamic equations describing Darwin selection process, being demonstrated here by the simple examples of the DSs, leads 
to an interesting, by our opinion, aspects of the dynamics. For example, DS can be investigated in the framework of near-integrable Hamiltonian systems with more complicated functions $\mu_{i}, D$ then studied in the present work.

It is pertinent to note that in the present work the phase space $\mathcal{M}$ includes original information space $\mathcal{A}$. Such way of Hamiltonization seems to be more preferable if compare to one proposed in [18] for the Volterra-Lotka type systems where phase space is built up from the original variables. In the latter case a number of constraints are imposed on the system that restricts the generality. 


\section{REFERENCES}

[1] Timofeeff-Ressovsky N.W. In: The Biosphere Meditations. (Ed. by Russian Academy of Natural Sciences, Moscow, 1996). p. 77.

[2] Eigen M., Schuster P. The Hypercycle. A Principle of Natural SelfOrganization (Springer-Verlag, Berlin, Heidelberg, New York, 1979). p. 270.

[3] Pechurkin N.S. Energy aspects of over-organismic systems development (Nauka, Novosybirsk, 1982). p. 113.

[4] Feistel R., Ebeling W. Biosystems 15 (1982) 291.

[5] Fisher R.A. The genetical theory of natural selection (Clarendon Press, Oxford, 1930).

[6] Zoglin L.N., Vladimirova M.G., Semenenko V.E. Plant Psysiology 17 (1970) 1129. (in Russian).

[7] Jones B.L. Bulletin of Mathematical Biology 41 (1979) 449.

[8] Svirezhev Yu.M., Logofet D.O. Stability of Biological associations (Nauka, Moscow, 1978).

[9] Svirezhev Yu.M., Pasekov V.P. Principles of Mathematical genetics (Nauka, Moscow, 1982).

[10] Pykh Yu.A. Equilibrium and Stability in Population Dynamics Models (Nauka, Moscow, 1983). 
[11] Feistel R., Ebeling W. In: Thermodynamics and Regulation of biological processes (Nauka, Moscow, 1984). p. 260.

[12] Ishida K. In: Thermodynamics and Regulation of biological processes (Nauka, Moscow, 1984). p. 238.

[13] Evdokimov E.V. In: Proc. of Intern. Conf. "Criteria of self- organization in physical, chemical and biological systems". (Moscow-Suzdal, 1995). p. 32.

[14] Shannon C.E., Weaver W. The Mathematical Theory of Communication (Urbana Univ. of Illinois Press, 1949).

[15] Pirt S.J. The bases of microorganism and cell cultivation (Mir, Moscow, 1978).

[16] Dykhuizen D., Hartl D. Evolution 35 (1981) 581.

[17] Dykhuizen D., Daniel E. Methods in enzymology 224 (1993) 613.

[18] Cronström C., Noga M. Multi-Hamiltonian structure of Lotka-Volterra and quantum Volterra models. Preprint hep-th/9412122. 